\title{
A New Method to Better Measure and Interpret Non- Verbal Communication in Patient-Therapist Interactions
}

\section{Felicitas Datz}

Medical University Vienna

\section{Guoruey Wong}

Universite de Montreal

Henriette Löffler-Stastka ( $\square$ henriette.loeffler-stastka@meduniwien.ac.at )

Medical University Vienna https://orcid.org/0000-0001-8785-0435

\section{Research note}

Keywords: psychotherapy, facial microexpressions, FACS, EmFACS, nonverbal communication, new methods, emotions

Posted Date: April 23rd, 2020

DOl: https://doi.org/10.21203/rs.3.rs-23084/v1

License: (c) (i) This work is licensed under a Creative Commons Attribution 4.0 International License. Read Full License 


\section{Abstract}

Objective(s): To develop a novel method of examining facial affects in psychotherapeutic settings and interpreting the subliminal microexpressions and emotions they represent.

Results: Therapy sessions are videotaped, and verbal content is evaluated with computer assistance from ATLAS.ti. Visual content is screened for facial action units/micro-expressions using the Facial Action Coding System (FACS) and reevaluated using the Emotional Facial Action Coding System (EmFACS) in order to discern the underlying emotions. Working Alliance Inventory scores are compiled to assess their impact on the quality of the therapeutic relationship. Reliability of facial action unit coding is ensured by a training course and an independently evaluated standardized test at Innsbruck University. Interrater reliability was excellent (Cohen's $\mathrm{K}>0.80$ ). Application of this method on a small study population to measure non-verbal communication and affects demonstrated its feasibility and usability. We believe this methodology to be widely implementable. Its application in psychotherapy may provide greater insights in investigations into important aspects of therapeutic interaction (e.g. verbal/non-verbal communication between clinicians and patients in psychiatric contexts, quality of psychotherapeutic relationships, etc.), and may be exploitable in clinical psychotherapeutic settings to better understand mechanisms of change in therapy.

\section{Introduction}

Process-oriented research is an important part of modern psychotherapeutic research. Nevertheless, there remains a long way to go in order to fully understand psychotherapeutic processes in the treatment of mental illness. In particular, the investigation of so-called therapeutic micro-processes, small but essential aspects of the patient - therapist interaction (especially facial micro-expressions), could bring promising insight into the mechanism of psychotherapy, as well as allow identification of specific factors essential to successful psychotherapy, regardless of the types of psychotherapeutic methods used.

Studies show that $60-80 \%$ of all communication (in treatment) is nonverbal $(1,2)$. Many nonverbal events are unconscious and often convey a patient's emotional and psychological state in a way that verbal communication cannot (3). In any given interpersonal interaction, a great number and variety of facial expressions are normally present (4); unfortunately, many of the visible expressions in conversations of two or more people are often inauthentic and very consciously controlled. Microexpressions, on the other hand, can neither be controlled nor be voluntarily displayed, and thus offer a truer view into the genuine feelings and emotions of a person. Discovered in the late 1960s by Paul Ekman, micro-expressions are defined as facial expressions of brief duration, lasting only for one-quarter to one-half of a second (5), and can be understood as either repressed or unconscious expression of emotion $(2,6)$. Ekman's research led him differentiate and name seven universal or basic emotions, expressible in the form of micro-expressions: Happiness, Surprise, Anger, Sadness, Fear, Disgust, and Contempt. In the context of psychotherapeutic interactions, the fact that micro-expressions are signals 
perceived unconsciously and can influence the patient-therapist relationship dynamic makes their investigation worthwhile.

What's more, therapy sessions with higher levels of nonverbal micro-affectivity have been rated as having more impact on patient outcome (7). If that is the case, it shows that common methods of analysis between cognition and affect might be incomplete, as they do not capture the speechless forms of affective expression. In addition, subconscious interaction patterns are implemented trough nonverbal signals. If the therapist is able to resist this relationship offer verbally as well as nonverbally, then pathological relationship patterns may be prevented, which in turn may increase the probability of successful treatment (8).

In this light, it seems promising to investigate the association between verbal psychotherapeutic interventions and the involuntary facial micro-expressions provoked by them (9), especially as it pertains to the working alliance, as this may allow us to better evaluate the effectiveness of certain interventions in therapeutic settings. Here below, we describe our novel method of how to measure and evaluate these micro-processes, with some preliminary findings obtained by applying them on a small sample study population.

\section{Our Methodology Study Population}

Our methods were applied on 20 willingly participating adult patients (10 males, 10 females; mean age $=$ 50.25 years) being treated in the Psychiatry Department and/or the Clinic for Psychoanalysis and Psychotherapy of Kaiser-Franz-Josef Hospital in Vienna, and 7 therapists (2 males, 5 females) treating them. Patients and their treating therapists needed to consent in order to participate and gave their written informed consent. The ethics committees of both the Medical University of Vienna and Vienna General Hospital approved this study.

\section{Video Recording}

Videotaped therapy sessions were held between the patient and the therapist alone. Both therapist and patient were recorded with separate video cameras, providing front views of each, using two high definition $4 \mathrm{k}$ cameras, each recording sixty frames per second. This is necessary in order to be able to detect the very subtle facial movements producing micro-expressions. In order to randomize the material, the second five minutes of each recorded session are analyzed with a split-screen technique.

\section{Verbal Content Analysis}

Following videotaping, sessions are transcribed, and their verbal content evaluated ("content analysis") according to the Psychodynamic Intervention List (PIL) (10), an instrument used to identify and rate psychodynamic verbal techniques, with computer assistance from ATLAS.ti. To increase confidence in the findings, self- and observer- rated measures are combined before and after the sessions. 
ATLAS.ti is software developed for systematic analysis and structuring of unstructured data, not only in text-form, but also multimedia files, such as videos. This was very conducive to this project as all four raters were able to feed videos of recorded sessions into the software and then analyze the transcripts while watching the playback, thus allowing the inclusion of atmospheric and contextual facets of the interaction.

This analysis was performed in order to capture verbal statements within interactions and categorize them, with a focus on identifying potential patterns in patient or therapist micro-expressions in association with certain subjects or types of verbal interactions. Through this process, qualitative data can be transformed into quantitative units, allowing for a reduction of complexity and thus facilitating comparisons.

\section{FACS + EmFACS Analysis / Coding}

The second phase of analysis consists of analyzing the visual content for action units using the Facial Action Coding System (FACS) (11). FACS is a coding system, associating facial expressions with socalled Action Units (AU) and Action Descriptors (AD). AUs are defined as the basic actions of individual muscles or groups of muscles, whereas ADs are described as unitary movements and actions of several muscle groups working together.

The coding is performed following a complex set of rules, outlined in (12). In essence, the authors divide the face into different parts and different rating units. In total, there exist twenty-five different action units that can emerge individually or in combinations, with differing positions of the head and eyes, and varying degrees of deceptive activity. Additionally, the intensity of the displayed action units is scored. The intensity scale ranges from $A-E$, with $A$ indicating minimal trace activity of the action unit, up to $E$, which indicates maximal intensity.

To perform the FACS coding, the videos of therapeutic sessions were assessed with split screen after having identified the different types of interventions and rating them using PIL. First, the videos were displayed together, and then separately at original speed. Following the guidelines of Ekman et al. regarding the Facial Action Coding System, the lower, middle, and upper facial regions were examined sequentially, in original time and then in slow-motion (10\%) using the coding sheet. The results are combinations of action units at different time points of the interaction.

Following that, the coded action units are then reevaluated using the Emotional Facial Action Coding System (EmFACS) (13) and Cohn-Kanade (CK+) datasets in concert (14). EmFACS is simply a system for classifying facial expressions that have been previously coded into so-called action units by the Facial Action Coding System. Using the FACS codes, the EmFACS instrument partitions the scored facial movements into groups that representation affective display. The coding must only be performed by FACS-certified raters, as according to Ekman and his group, only trained raters are qualified to perform EmFACS rating. We complemented our evaluation with the very popular Extended CK + Dataset (14), since 
this dataset is generally considered as being more comprehensive than the basic one that EmFACS traditionally uses, with many more AU combinations and associated emotions available.

\section{Validation}

The reliability of all coding was ensured by a training course at the Study Group of Prof. Eva BänningerHuber from Innsbruck University, followed by a standardized final test, which was independently evaluated by the team of Paul Ekman. Interrater reliability of the final test was very good (Cohen's K > $0.80)$.

Raters conducting the content analysis and the analysis of the Facial Action Coding System and EmFACS were all blinded to the patients' diagnoses, as well as the therapists' theoretical background and experience.

\section{Working Alliance}

Finally, the results of the Working Alliance Inventory were evaluated via the Working Alliance InventoryShort Revised (WAI-SR), which measures the quality of the patient-therapist working relationship by assessing three key aspects: (a) agreement on the tasks of therapy, (b) agreement on the goals of therapy and (c) development of an affective bond. (15). Studies revealed that working alliance quality has a significant impact on treatment outcome and can even be used as a predictor of success (16-18).

\section{Statistical Analysis}

Statistical analyses were performed using the IBM SPSS Statistics 19 software (IBM Corp., Armonk, NY, USA). For all analyses, the significance threshold was defined as $p \leq 0.05$.

Differences between therapists and patients with respect to facial affects analysed via FACS were assessed with Mann-Whitney's U test. The standardized mean group difference, Cohen's $d$, and the conventional benchmarks for interpretation (small effect: $r \leq .10$, medium effect: $r \leq .30$, large effect: $r$ $\leq .50$; [33]) were applied.

\section{Results}

Overall, the group of patients displayed significantly fewer facial affects than the group of therapists (Mann-Whitney-U $=146.0, p=0.023, d_{\text {Cohen }}=0.72$ ). However, there was no significant difference regarding the variety of different facial affects between therapists and patients (Mann-Whitney-U $=199.5, p=0.304$, $\mathrm{d}_{\text {Cohen }}=0.308$ ). Table 1 shows the frequency of each basic affect in patients and therapists. The most frequently occurring affects in patients were disgust and surprise; in therapists, contempt and surprise. Since in both groups, fear, anger, and sadness affects were only marginally present, for simplicity these three were grouped together under term "else". 
Table 1. Facial affect display of patients and therapists.

\begin{tabular}{lccccc}
\hline & \multicolumn{2}{c}{ Patients } & & \multicolumn{2}{c}{ Therapis ts } \\
\cline { 2 - 3 } \cline { 5 - 6 } & mean & SD & & mean & SD \\
\hline Joy & 0,41 & 0,734 & & 0,86 & 1,959 \\
Contempt & 0,50 & 0,740 & & 1,45 & 1,371 \\
Disgust & 0,82 & 1,053 & & 0,55 & 1,224 \\
Surprise & 0,68 & 1,211 & & 3,18 & 5,170 \\
Else & 0,05 & 0,213 & & 0,09 & 0,294 \\
\hline
\end{tabular}

\section{Discussion}

The methodology outlined in this paper was developed and refined at Vienna General Hospital, in what could be considered a typical social psychiatric ward. This department provides everyday psychiatric hospitalization services to those who need them, offering coverage to the roughly 200,000 Austrian citizens living within its service radius. The patients encountered there on a daily basis are extremely diverse in many aspects, as much from a demographic point of view as from a medical one. Many times, this diversity poses great challenges in communicating with, handling, and/or treating these patients. Often times, for example, a patient may not speak the same language as the treating personnel or staff, or perhaps the patient's medical condition (psychiatric or otherwise), level of cognitive function or even literacy hinders verbal communication. In other scenarios as well, for whatever reason, there may be concealment or untruthfulness (either wilfully or inadvertently) in verbal interactions or in responding to typical clinical self-report questionnaires items. In such cases, the patient-clinician relationship will likely be less than ideal, rendering treatment difficult, and at times, perhaps even dangerous. Tools that would allow monitoring patient affects more closely and accurately at all phases of a patient's therapeutic experience (from admission and during treatment, to discharge) would be a significant advance, potentially improving patient outcomes and security of all parties involved in treatment.

Overall, the methods developed and presented in this work are extremely feasible, adaptable, and powerful tools that may prove very useful across a wide variety of clinical contexts: treatment progression monitoring, to be sure, but also for enhancing clinical training of practitioners and the patient-clinician working relationship. In so doing, this may serve to improve psychotherapeutic treatment outcomes in the long-term.

\section{Limitations}

Some limitations should be noted. Firstly, EmFACS can only detect very primary or basic emotions, so at times the instrument fails to distinguish nuances of displayed primary affects, even when complemented with the $\mathrm{CK}+$ dataset. This task is thus left to the interpreters of the EmFACS results, with a high degree of subjectivity involved in the evaluation. In addition, if one is limited to EmFACS, it is difficult to discern what complex and multifaceted dimensions or meanings any discernible emotion might have for the 
interacting partners, especially when it comes to unconscious understanding. Finally, the complexity and meticulousness of the rating methods makes it quite demanding (time-consuming), as evaluating even one minute of a video interview corresponds to roughly five hours of work, in order to process and rate the material contained therein.

Ideally, the validity of the coding of emotions obtained through EmFACS should be verified by a comparison of its findings against those obtained through other means (preferably one considered as being a "gold-standard"). Further refinement of these techniques is urgently needed to gather real-world data and address the major limitations we have identified at present, in order to increase the scope of their applicability as well as reduce the time and resource demands.

\section{Abbreviations}

Action Descriptors

$A D$

Action Units

$\mathrm{AU}$

Affect Experience and Affect Regulation Q-Sort Test

AREQ

Cohn-Kanade

$\mathrm{CK}+$

Emotional Facial Action Coding System

EmFACS

Facial Action Coding System

FACS

Psychodynamic Intervention List

PIL

Working Alliance Inventory-Short Revised

WAI-SR

\section{Declarations}

Ethics approval and consent to participate: The ethics committee of the Medical University of Vienna approved the study (EK-Votum 2153/2015). Participants gave their written informed consent.

Consent for publication: Not applicable.

Availability of data and material: The datasets generated and analyzed during the current study are not publicly available due to reasons of confidentiality according to the ethics committee of the Medical University of Vienna but are available from the corresponding author upon reasonable request.

Competing interests: All authors declare that they have no conflict of interest. 
Funding: Not applicable.

Authors' contributions: HLS and FD conceived the study, managed and organized the data, gave feedback to the design, repeatedly read and finalized the paper. GW read the paper repeatedly, edited, redrafted, and organized the paper for final submission. All authors read and approved this manuscript.

Acknowledgements: The authors would like to thank Claudia Zederbauer for rating the video material, and Prof. Bänninger-Huber for certification of the facial action coding course.

\section{References}

1. Burgoon JK, Guerrero LK, Floyd K. Nonverbal communication: Routledge; 2016.

2. Rasting $M$, Beutel ME. Dyadic affective interactive patterns in the intake interview as a predictor of outcome. Psychotherapy Research. 2005;15(3):188-98.

3. Philippot P, Feldman RS, Coats EJ. Nonverbal behavior in clinical settings: Oxford University Press; 2003.

4. Scherer KR. Appraisal considered as a process of multilevel sequential checking. Appraisal processes in emotion: Theory, methods, research. 2001;92(120):57.

5. Haggard EA, Isaacs KS. Micromomentary facial expressions as indicators of ego mechanisms in psychotherapy. Methods of research in psychotherapy: Springer; 1966. p. 154-65.

6. Freitas-Magalhães A. Facial expression of emotion. 2012.

7. Anstadt T, Merten J, Ullrich B, Krause R. Affective dyadic behavior, core conflictual relationship themes, and success of treatment. Psychotherapy Research. 1997;7(4):397-417.

8. Datz F, Parth K, Rohm C, Madanoglu S, Seidman C, Löffler-Stastka H. Dimensions of activity in countertransference and therapist reactions: Therapist reactions during sessions with depressed patients. Zeitschrift für Psychosomatische Medizin und Psychotherapie. 2016;62(4):322-35.

9. Alves C, Freitas-Magalhães A, Bluhm C, Davis M, Sullivan G, Montoya D, et al. Handbook on Facial Expression of Emotion2013.

10. Gumz A, Horstkotte JK, Kästner D. Das Werkzeug des psychodynamischen Psychotherapeutenverbale Interventionstypen aus theoretischer und aus der Praxis abgeleiteter Perspektive. Zeitschrift für Psychosomatische Medizin und Psychotherapie. 2014;60(3):219-37.

11. Ekman P, Friesen WV. Manual for the facial action coding system: Consulting Psychologists Press; 1978.

12. Ekman P, Friesen WV, Hager JC. Facial action coding system: The manual on CD ROM. A Human Face, Salt Lake City. 2002:77-254.

13. Friesen W, Ekman P. EMFACS-7: Emotional facial action coding system, Version 7. Unpublished manual. 1984.

14. Lucey P, Cohn JF, Kanade T, Saragih J, Ambadar Z, Matthews I, editors. The extended cohn-kanade dataset (ck+): A complete dataset for action unit and emotion-specified expression. 2010 IEEE 
Computer Society Conference on Computer Vision and Pattern Recognition-Workshops; 2010: IEEE.

15. Munder T, Wilmers F, Leonhart R, Linster HW, Barth J. Working Alliance Inventory-Short Revised (WAISR): psychometric properties in outpatients and inpatients. Clinical psychology \& psychotherapy. 2010;17(3):231-9.

16. Barber J, Connolly M, Crits-Christoph P, Gladis L, Siqueland L. Alliance predicts patients' outcome beyond in-treatment change in symptoms. Personality Disorders: Theory, Research, and Treatment. 2009;S:80-9.

17. Krupnick JL, Sotsky SM, Elkin I, Simmens S, Moyer J, Watkins J, et al. The role of the therapeutic alliance in psychotherapy and pharmacotherapy outcome: Findings in the National Institute of Mental Health Treatment of Depression Collaborative Research Program. Focus. 2006;64(2):532-277.

18. Leichsenring F. Are psychodynamic and psychoanalytic therapies effective?: A review of empirical data. The International Journal of Psychoanalysis. 2005;86(3):841-68. 